\title{
Acupuntura e o tratamento da ansiedade: Revisão sistemática de literatura
}

\author{
Acupuncture and the treatment of anxiety: Systematic literature review
}

Vinicius Macêdo de Sousa ${ }^{1}$

\section{RESUMO}

Baseada no princípio de que o homem deve estar em harmonia com a natureza, a acupuntura é um dos muitos elementos que compõem a Medicina Tradicional Chinesa. A acupuntura vem se destacando como forma de tratamento complementar, tanto para ansiedade como para diversas patologias. O objetivo deste trabalho é avaliar as evidências científicas disponíveis na literatura sobre os efeitos da acupuntura no tratamento da ansiedade e a qualidade desses estudos entre os anos de 2010 a 2020. Este trabalho se fundamentou em revisão sistemática da literatura científica nacional e internacional sobre ansiedade e acupuntura nas produções científicas publicadas em periódicos indexados nos bancos de dados da Literatura Latino Americana e do Caribe em Ciências da Saúde (LILACS), da Scientific Electronic Library Online (SciELO) e da United States National Library of Medicine (PubMed). De um modo geral, os tratamentos da Medicina Tradicional Chinesa trazem mais benefícios nos grupos estudados, principalmente nos experimentos de longo prazo, demonstrando ser esta uma possibilidade terapêutica para o tratamento de pessoas com ansiedade. Apesar dessas descobertas, fazem-se necessários mais estudos clínicos e sistematizados, com amostras maiores através de pesquisas básicas e clínicas para mostrar a eficácia ou não desses procedimentos.

Palavras-chave: Acupuntura. Ansiedade. Revisão Sistemática de Literatura.

\section{ABSTRACT}

Based on the principle that man must be in harmony with nature, acupuncture is one of the many elements that make up Traditional Chinese Medicine. Acupuncture has stood out as a form of complementary treatment, both for anxiety and for various pathologies. The objective of this work is to evaluate the scientific evidence available in the literature on the effects of acupuncture in the treatment of anxiety and the quality of these studies between the years 2010 to 2020. This work was based on a systematic review of the national and international scientific literature on anxiety and acupuncture in scientific productions published in journals indexed in the databases of Latin American and Caribbean Literature in Health Sciences (LILACS), the Scientific Electronic Library Online (SciELO) and the United States National Library of Medicine (PubMed). In general, Traditional Chinese Medicine treatments bring more benefits to the groups studied, especially in long-term experiments, demonstrating that this is a therapeutic possibility for the treatment of people with anxiety. Despite these discoveries, more clinical and systematic studies are needed, with larger samples through basic and clinical research to show the effectiveness or not of these procedures.

Keywords: Acupuncture. Anxiety. Systematic Literature Review.
Graduação em Farmácia Generalista (ITPAC Araguaína), pós-graduando em Acupuntura (IEES) e Técnico em Farmácia no Hospital de Doenças Tropicais HDT-UFT - EBSERH.

\section{E-mail:}

viniciusmsfarm@gmail.com 


\section{INTRODUÇĀO}

O termo ansiedade é usado para descrever a resposta mental e psíquica nos indivíduos a situações que despertam medo ou ameaça. Dentre as respostas do corpo humano a esses impactos podem-se incluir tremores, falta de ar, aumento dos batimentos cardíacos, sudorese, alterações gastrointestinais entre outros.

A ansiedade na ciência ocidental é considerada uma resposta fisiológica natural, causando os sintomas de taquicardia, sentimento de medo generalizado, medo de desastre eminente, tensão e inquietação. Esses sintomas também podem estar relacionados a doenças, como fobias, síndrome do pânico, transtornos obsessivo-compulsivos, ansiedade generalizada, entre outros ${ }^{1}$.

A ansiedade há muito já foi identificada na ciência ocidental e bastante estudada pelas áreas da Psicologia, da psicanálise e da Medicina. A ansiedade não é considerada um fenômeno necessariamente patológico, e é mais bem entendida como uma função natural do organismo que permite que o mesmo esteja preparado ou que se prepare para responder da melhor forma possível a uma situação nova e desconhecida ou a uma situação já conhecida e interpretada como potencialmente perigosa. Entretanto, se a ansiedade atingir graus muito elevados e contínuos, ela pode ser considerada prejudicial ao organismo, pois fará com que este permaneça em constante estado de alerta, configurando então, uma situação patológica².

A Organização Mundial da Saúde (OMS) divulgou pesquisa em 2017, em que o Brasil aparece como o país com maior taxa de depressão na América Latina e que a prevalência de Transtorno Mental Comum-TMC na população geral é de 29,9\%, podendo chegar aos $38 \%$ dos usuários da Atenção Primária à Saúde. E, no contexto mundial, a OMS aponta que 322 milhões de pessoas sofrem com depressão, dados esses que correspondem a $4,4 \%$ da população mundial, sendo ainda um número $18 \%$ a mais do que em 2007 , ou seja, do que há 10 anos $^{3,4}$.

"Geralmente a população acaba optando pelos tratamentos convencionais, como é o caso dos medicamentos ansiolíticos, entretanto, estes podem acarretar problemas como dependência medicamentosa" 5 . Sendo assim, nas últimas décadas, a acupuntura vem se destacando como forma de tratamento complementar, tanto para ansiedade como para diversas patologias. Contudo, ainda não há estudos suficientes relacionados à eficácia deste tratamento em indivíduos com ansiedade. 
"No Brasil, a Acupuntura foi introduzida há cerca de 40 anos. Em 1988, por meio da Resolução № 5/88, da Comissão Interministerial de Planejamento e Coordenação (Ciplan), teve as suas normas fixadas para o atendimento nos serviços públicos de saúde" ${ }^{\text {. S Segundo }}$ a Política Nacional de Práticas Integrativas e Complementares (PNPIC), que foi aprovada no Sistema Único de Saúde (SUS) pela portaria 971/2006 do Ministério da Saúde, em que a prática de acupuntura pode ser realizada por profissionais de saúde que tenham realizado o curso de especialização, a Medicina Tradicional Chinesa caracteriza-se por um sistema médico integral, originado há milhares de anos na China. E, a acupuntura é um tipo de tecnologia de intervenção em saúde, originária dessa Medicina Tradicional Chinesa, que pode ser usada de maneira isolada ou associada a outros tipos procedimentos.

A acupuntura ainda, se baseia na estimulação de pontos anatômicos precisos do corpo, chamados de acupontos, definidos por meio da inserção de agulhas filiformes metálicas para auxiliar na promoção, manutenção e recuperação da saúde, assim como para prevenção de agravos e doenças. Ainda de acordo com o PNPIC ${ }^{6}$ (2006), de acordo com a Medicina Tradicional Chinesa, a acupuntura é uma técnica terapêutica milenar amplamente utilizada atualmente por profissionais capacitados na área da saúde. A acupuntura preconiza o tratamento das doenças através da normalização dos órgãos adoecidos.

A partir disso, tem-se que o objetivo geral deste trabalho é avaliar as evidências científicas disponíveis na literatura sobre os efeitos da acupuntura no tratamento da ansiedade e a qualidade desses estudos entre os anos de 2010 a 2020. Esse recorte temporal foi adotado uma vez que, durante esse período, no Brasil, essa terapia foi mais popularizada e passou a ser mais utilizada na prática clínica, incluindo o tratamento da ansiedade com aval da PNPIC.

A Medicina Tradicional Chinesa (MTC) baseia-se no taoísmo, movimento filosófico desenvolvido a partir da observação da natureza. Através de uma visão holística, vê o homem integrado ao seu meio ambiente, assim como a sua fisiologia.

Achados arqueológicos permitem supor que essa fonte de conhecimento remonta há pelo menos 3.000 anos. A denominação chinesa zhen jiu, que significa agulha (zhen) e calor (jiu) foi adaptados nos relatos trazidos pelos jesuítas no século XVII como Acupuntura (derivada das palavras latinas acus, agulha e punctio, punção). $O$ efeito terapêutico da estimulação de zonas neurorreativas ou "pontos de acupuntura" foi, a princípio, descrito e explicado numa linguagem de época, simbólica e analógica, consoante com a filosofia clássica chinesa ${ }^{6}$. 
Para Yamamura7 (2001), na Medicina Tradicional Chinesa não existe separação entre mente, corpo e espírito. Com isso, não há uma classificação de doenças ou distúrbios que sejam exclusivamente psicológicos ou psiquiátricos, pois tudo está interligado.

De acordo com Silva² (2010), os fenômenos como a ansiedade "são sintomas (assim como no ocidente) de distúrbios de outra ordem. Aliado a isso, como na MTC não existe separação entre corpo, mente e espírito, uma desarmonia em um dos cinco principais órgãos do corpo (na perspectiva chinesa: coração, baçopâncreas, pulmão, rins e fígado) ocasionará automaticamente um desequilíbrio nos aspectos mentais e espirituais desses órgãos, chamados respectivamente de shen, hun, po, yi e zhi".

Baseada no princípio de que o homem deve estar em harmonia com a natureza, a acupuntura é um dos muitos elementos que compõem a Medicina Tradicional Chinesa. A partir dos princípios yin e yang, opostos e complementares, eles compõem o universo e resultam no equilíbrio e na harmonia, gerando saúde. Quando alguma doença aparece no organismo gerando desequilíbrio que altera o qi e o xue, a acupuntura auxilia minimizando ou eliminando esse desequilíbrio, reestabelecendo o fluxo $0^{8,2,9}$.

Assim, a acupuntura tem como base o princípio básico do equilíbrio, em que ele "é mantido no corpo humano por meio do fluxo suave de uma energia denominada pelos chineses qi, bem como pelo fluxo, também suave, pelo corpo, do sangue, denominado pelos chineses como xue"2.

A acupuntura atua em todo o sistema nervoso e não trata apenas o local comprometido no corpo. Com isso, ela acaba por estimular o mecanismo de compensação e equilíbrio no corpo todo ${ }^{10}$.

Segundo Zotelli, Meirelles e Sousa"11 (2017), na Medicina Tradicional Chinesa "os pontos de acupuntura são a área mais externa do corpo energético do indivíduo, funcionando como elo de comunicação entre o meio interno e o externo". Esses pontos de acupuntura, podem ainda alterar a dinâmica da circulação sanguínea, gerar o relaxamento muscular, curando espasmos, atenuando a inflamação e a dor. Com a acupuntura ainda, o corpo libera hormônios como o cortisol e endorfinas.

O mecanismo de ação da acupuntura se dá através da introdução da agulha em pontos específicos dos meridianos energéticos, onde é gerado um estímulo nas terminações nervosas em nível dos músculos, que vai para o sistema nervoso central, onde é reconhecido e traduzido em três níveis: nível hipotalâmico, onde há ativação do eixo hipotálamo-hipófise que gera liberação de $\beta$-endorfinas (analgésicos), cortisol (antiinflamatório) e serotonina (antidepressivo) na corrente sanguínea e líquida encefálico 
raquidiana; no nível do mesencéfalo ocorrerá a ativação de neurônios da substância cinzenta, sendo liberada endorfinas e estas vão estimular a produção de serotonina e norepinefrina, já no nível de medula espinal, haverá a ativação de interneurônios na substância gelatinosa e liberação de dinorfinas. A entrada da agulha na pele provoca uma microinflamação que ativa a produção natural dessas substâncias. Através da liberação desses neurotransmissores, ocorre um bloqueio da propagação dos estímulos dolorosos, impedindo sua percepção pelo cérebro, desencadeando um processo de analgesia. Assim, a resposta do organismo é mais rápida, diminuindo e/ou fazendo desaparecer os sintomas $^{12}$.

Como exposto acima, a acupuntura vem sendo utilizada amplamente para tratamento de diversas patologias, inclusive ansiedade e depressão. Dessa forma, o tratamento em Medicina Tradicional Chinesa se divide em três momentos: a) Natureza ajudando o doente, em que se utiliza naturoterápicos na sua forma animal, vegetal ou mineral; b) Homem ajudando o doente, em que entram as terapias de acupuntura, Tui-Ná e/ou Qi Gong; e, c) o doente se ajudando, em que ele pratica exercícios que reestabelecem sua energia como Tai-Chi-Chuan, Tao-In (busca interna) e Chi Gong (ou Qi Gong) ${ }^{13}$.

Segundo Ross (2003) ${ }^{14}$, a ansiedade surge a partir de um desequilíbrio entre os sistemas cardiovascular e o renal, ou seja, os sistemas do coração e do rim: Sentimentos de apreensão, do medo de que algo terrível aconteça são característicos da ansiedade, que pode vir combinada de sobressaltos e receio, com sinais físicos como tremor, frequência urinária ou intestinos soltos.

Logo, o autor afirma ainda que a ansiedade é o resultado de um distúrbio do shen, em que indica que o espírito não está se movendo adequadamente pelo corpo. Para Ross $(2003)^{14}$, existem pelo menos três tipos diferentes de ansiedade, de acordo com a situação que a originou:

[...] Ansiedade por excesso: [...] O fogo fleuma do coração é uma forma de excesso que pode levar à ansiedade e à confusão de pensamento, linguagem e comportamento. consiste, essencialmente, em fleuma, decorrente da deficiência do baço, em combinação com o fogo do coração. Pode surgir de um estresse emocional ou do excesso de fumo, do álcool e de alimentos gordurosos, com falta de exercícios físicos. [...] Ansiedade por estagnação: A estagnação pode dar origem ao distúrbio do movimento. A estagnação do qi do coração e do qi do fígado, por exemplo, decorrentes da estagnação emocional, podem levar ao distúrbio do espírito do coração e à hiperatividade do yang do fígado, levando à ansiedade. A estagnação do qi pode resultar em acúmulo de fleuma, que pode perturbar a 
livre circulação do espírito, causando ansiedade. [...] Ansiedade por deficiência: [...] a ansiedade aumenta quando a energia está reduzida, quando há deficiência por falta de sono e descanso, excesso de trabalho, estresse, doença e nutrição deficiente, além de outros fatores. A deficiência do qi do coração e do rim, do yin do coração e do rim, e do sangue do coração e do baço podem dar origem à ansiedade, já que o qi, o yin e o sangue são necessários para manter o espírito estável (ROSS, 2003, p. 465) ${ }^{14}$. (grifo nosso).

Alguns dos principais pontos utilizados no tratamento do transtorno de ansiedade generalizada e suas funções. De acordo com Focks ${ }^{15}$ (2005) e Ross ${ }^{14}$ (2003), os pontos relacionados na Tabela 1, têm as seguintes funções dentro do tratamento de ansiedade, a partir da acupuntura:

Tabela 1. Pontos da acupuntura para tratamento da ansiedade.

\begin{tabular}{|c|c|c|}
\hline Ponto & Local & Função \\
\hline R3 & $\begin{array}{l}\text { terceiro ponto do canal de } \\
\text { energia do rim }\end{array}$ & $\begin{array}{l}\text { Regula o equilíbrio do yin e do yang, fortalece e estabiliza a } \\
\text { mente e as emoções, equilibra a labilidade emocional, a } \\
\text { deficiência do qi do rim; tonifica o rim e beneficia a essência e } \\
\text { tonifica o sangue; }\end{array}$ \\
\hline R6 & $\begin{array}{l}\text { sexto ponto do canal de } \\
\text { energia do rim }\end{array}$ & $\begin{array}{l}\text { Tonifica o yin do rim, a deficiência do rim, nutre o yin, } \\
\text { principalmente quando existe excesso de fogo no coração, } \\
\text { promove o sono e os fluidos corpóreos; }\end{array}$ \\
\hline $\mathrm{C7}$ & $\begin{array}{l}\text { sétimo ponto do canal de } \\
\text { energia do coração }\end{array}$ & $\begin{array}{l}\text { Tonifica o coração, equilibra o yin e o yang, estabiliza o } \\
\text { coração, clareia a mente, acalma a mente e as emoções, } \\
\text { regula o espírito, tonifica o sangue, tonifica o yin do coração, } \\
\text { elimina o fogo; }\end{array}$ \\
\hline CS6 & $\begin{array}{l}\text { sexto ponto do canal de } \\
\text { energia } \\
\text { coração/sexualidade }\end{array}$ & $\begin{array}{l}\text { Move a estagnação e acalma irregularidades do qi, remove a } \\
\text { estagnação de sangue e fleuma, acalma o espírito, remove a } \\
\text { estagnação do qi do pulmão, tonifica o coração; é indicado } \\
\text { para dor, choque e traumatismo; }\end{array}$ \\
\hline CS7 & $\begin{array}{l}\text { sétimo ponto do canal de } \\
\text { energia } \\
\text { coração/sexualidade }\end{array}$ & $\begin{array}{l}\text { Acalma o espírito, move a estagnação e regula o qi do coração } \\
\text { e do estômago; }\end{array}$ \\
\hline E25 & $\begin{array}{l}\text { vigésimo quinto ponto do } \\
\text { canal de energia do } \\
\text { estômago }\end{array}$ & Regula o estresse emocional, regulariza o qi; \\
\hline E36 & $\begin{array}{l}\text { trigésimo sexto ponto do } \\
\text { canal de energia do } \\
\text { estômago }\end{array}$ & $\begin{array}{l}\text { Fortalece o baço e o estômago para produzirem qi e sangue, } \\
\text { que eliminam a umidade; faz subir o qi, tonifica o sangue e o } \\
\text { qi, estabiliza a mente e as emoções, regulariza o qi defensivo } \\
\text { e nutritivo; }\end{array}$ \\
\hline P5 & $\begin{array}{l}\text { quinto ponto do canal de } \\
\text { energia do pulmão }\end{array}$ & $\begin{array}{l}\text { Resfria e acalma o pulmão, trata a retenção de fleuma no } \\
\text { pulmão, trata a deficiência de yin no pulmão; }\end{array}$ \\
\hline P7 & $\begin{array}{l}\text { sétimo ponto do canal de } \\
\text { energia do pulmão }\end{array}$ & $\begin{array}{l}\text { Expele o vento externo, fortalece o pulmão melhorando a } \\
\text { circulação do qi defensivo, remove a estagnação do qi do } \\
\text { pulmão, remove as emoções estagnadas do pulmão, como a } \\
\text { tristeza e a mágoa reprimidas; }\end{array}$ \\
\hline P9 & $\begin{array}{l}\text { nono ponto do canal de } \\
\text { energia do pulmão }\end{array}$ & $\begin{array}{l}\text { Tonifica o qi do pulmão, tonifica o yin do pulmão, fortalece os } \\
\text { vasos sanguíneos e a circulação do sangue; }\end{array}$ \\
\hline IG4 & $\begin{array}{l}\text { quarto ponto do canal de } \\
\text { energia do intestino grosso }\end{array}$ & $\begin{array}{l}\text { Remove o vento exterior, remove o calor, relaxa a tensão } \\
\text { muscular, move estagnações do sangue, acalma a } \\
\text { hiperatividade do yang do fígado, acalma a mente, tonifica o qi } \\
\text { e o sangue; }\end{array}$ \\
\hline IG11 & $\begin{array}{l}\text { décimo primeiro ponto do } \\
\text { canal de energia do intestino } \\
\text { grosso }\end{array}$ & $\begin{array}{l}\text { Expele o vento exterior, remove o calor, relaxa a tensão } \\
\text { muscular e alivia a dor, acalma a hiperatividade do yang do } \\
\text { fígado, resolve a umidade; }\end{array}$ \\
\hline F3 & $\begin{array}{l}\text { terceiro ponto do canal de } \\
\text { energia do fígado }\end{array}$ & $\begin{array}{l}\text { Move a estagnação do qi e do sangue, acalma a hiperatividade } \\
\text { do yang do fígado, elimina o vento do fígado e reduz espasmos } \\
\text { e dor; tonifica o sangue e acalma o espírito; }\end{array}$ \\
\hline
\end{tabular}


F14 décimo quarto ponto do canal de energia do fígado

VC4 quarto ponto do canal de energia do vaso da concepção

vc6 sexto ponto do canal de energia do vaso da concepção

VC12 décimo segundo ponto do canal de energia do vaso da concepção

VC15 décimo quinto ponto do cana de energia do vaso da concepção

VC17 décimo sétimo ponto do canal de energia do vaso da concepção

BP3 terceiro ponto do canal de energia do baço-pâncreas

BP6 sexto ponto do canal de energia do baço-pâncreas

BP9 nono ponto do canal de energia do baço-pâncreas

TA4 quarto ponto do canal de energia do triplo aquecedor

TA5 quinto ponto do canal de energia do triplo aquecedor

Yintang ponto extra localizado no entre as sobrancelhas
Move a estagnação do qi do fígado, elimina a umidade calor do fígado; utilizado para congestionamento mental e emocional;

Fortalece o jing, o qi, o yin e o yang do rim, dispersa a estagnação do qi;

Tonifica a deficiência e move a estagnação do qi;

harmoniza a preocupação e a insegurança, tonifica a deficiência do qi e do yang do baço, move a estagnação e regula a rebelião do qi do estômago;

Acalma o espírito quando este está agitado pelo fogo do coração ou obstruído pela fleuma no coração;

Dispersa a estagnação do qi, remove a estagnação do qi do coração, do sangue do coração, do qi do pulmão e do aquecedor superior;

move a estagnação do qi do baço, tonifica a deficiência e fortalece o baço, resolve o esgotamento e o embotamento mental por umidade e fleuma;

tonifica o baço, o qi e o sangue, elimina a umidade, tonifica o yin, acalma a mente, regula o qi do fígado;

elimina a umidade;

tonifica a deficiência do qi do rim, remove o excesso do vento e calor e a estagnação do qi;

remove vento e calor e a estagnação do qi do fígado

Acalma a mente, diminui cefaleia, tonturas e a sensação de peso na cabeça; é utilizado em casos de estados de ansiedade, de distúrbios do sono e em estados de confusão mental.

Fonte: Focks ${ }^{15}$ (2005) e Ross ${ }^{14}(2003)$

Assim sendo, conforme apontado pelos autores, a partir desses protocolos usados na terapia de acupuntura, houve efeitos positivos no tratamento dos distúrbios da ansiedade.

\section{MATERIAIS E METODOS}

As etapas do desenvolvimento dessa revisão sistemática de literatura foram: 1) identificação do tema e seleção da questão de investigação de pesquisa; 2) estabelecimento de critérios de inclusão e exclusão de artigos (busca da literatura); 3) definição das informações a serem extraídas dos estudos selecionados; 4) avaliação dos estudos selecionados; e, 5) análise e síntese dos resultados e apresentação da revisão. Como questão norteadora desta revisão, tem-se o seguinte questionamento: Quais são as evidências científicas disponíveis na literatura, nos últimos 10 anos (2010-2020), sobre o efeito da acupuntura no tratamento da ansiedade?

Este trabalho se fundamentou em revisão sistemática da literatura científica nacional e internacional sobre ansiedade e acupuntura, com a finalidade de buscar propostas de intervenções e tratamentos da doença por meio dessa técnica, cujo objeto de estudo foi a 
produção científica publicada em periódicos indexados nos bancos de dados da Literatura Latino Americana e do Caribe em Ciências da Saúde (LILACS), da Scientific Electronic Library Online (SciELO) e da United States National Library of Medicine (PubMed).

Foi utilizado o operador booleano AND, formando assim, a estratégia de busca a partir dos descritores e termos controlados, com a seguinte combinação: anxiety AND acupuncture therapy AND acupuncture. A busca dos documentos ocorreu nos meses de janeiro a março de 2021.

Por meio da pesquisa, inicialmente foram rastreados 689 documentos, sendo que 652 foram encontrados na base de dados PubMed; e os demais, nas bases LILACS (30) e SciELO (7). Em seguida, foram selecionados os trabalhos científicos incluídos no estudo por meio de avaliação dos títulos, resumos e conclusões de forma independente, utilizando os seguintes critérios de inclusão: publicações realizadas nos últimos 10 anos; publicações com acesso aberto; publicados nos idiomas português, inglês ou espanhol; e, com o tema tratando sobre o tratamento da ansiedade por meio da acupuntura. Como critérios de exclusão, foram utilizados artigos fora da faixa de 10 anos de publicação; artigos com acesso pago e artigos que abordavam outras doenças além da ansiedade.

Posteriormente à avaliação realizada dos títulos, resumos e conclusões foram incluídos para análise nesta revisão sistemática de literatura 169 trabalhos, sendo que destes, após leitura dos resumos e aplicados os critérios de exclusão desta pesquisa, apenas 31 foram incluídos na mesma e 138 artigos excluídos devido os critérios da revisão. Sendo que desses, estão distribuídos: 2 na SciELO, 21 na PubMed e 8 na LILACS.

\section{RESULTADOS E DISCUSSÃO}

Para melhor compreensão e visualização dos resultados da análise dos artigos encontrados na revisão sistemática de literatura (RSL), os mesmos foram sistematizados e estão apresentados na forma de um quadro, com as seguintes especificações: autor, ano de publicação, título do artigo, tipo de estudo e objetivo. No tipo de estudo, identificamos conforme o autor especificou no trabalho. Vale ressaltar, que três artigos foram encontrados em duas bases de dados distintas, os quais serão identificados por um asterisco $\left({ }^{*}\right)$.

Tabela 2. Artigos analisados por meio da RSL entre os anos de 2010 a 2020.

\begin{tabular}{ccccc}
\hline Autores & Ano & Título & $\begin{array}{c}\text { Tipo de } \\
\text { Estudo }\end{array}$ & Objetivo \\
\hline Silva, ALP & 2010 & $\begin{array}{c}{ }^{*} \text { O tratamento da } \\
\text { ansiedade por }\end{array}$ & $\begin{array}{c}\text { Estudo de } \\
\text { Caso }\end{array}$ & $\begin{array}{c}\text { Relatar o tratamento realizado } \\
\text { por meio da acupuntura a }\end{array}$
\end{tabular}




\begin{tabular}{|c|c|c|}
\hline & & $\begin{array}{c}\text { intermédio da } \\
\text { acupuntura: um estudo } \\
\text { de caso. }\end{array}$ \\
\hline $\begin{array}{c}\text { Santos, DR } \\
\text { Sperotto, DF } \\
\text { Pinheiro, UMS }\end{array}$ & 2011 & $\begin{array}{c}\text { A Medicina Tradicional } \\
\text { Chinesa no tratamento } \\
\text { do transtorno de } \\
\text { ansiedade: um olhar } \\
\text { sobre o stress }\end{array}$ \\
\hline
\end{tabular}

Errington-Evans, $\mathrm{N}$

Bussell, J

Arvidsdotter, $T$

Taft, C

Moura, CC

Carvalho, CC

Silva, AM

lunes, $\mathrm{DH}$

Carvalho, EC

Chaves, ECL

Arvidsdotter, $\mathrm{T}$

Marklund, B

Taft, C
Marklund, B

2011 Acupuncture for Anxiety

The Effect of

Acupuncture on

Working Memory and Anxiety

Effects of an integrative treatment, therapeutic acupuncture and conventional treatment in alleviating

psychological distress in primary care patients - a pragmatic randomized controlled trial

Auriculoterapia efeito sobre a ansiedade

Six-month effects of integrative treatment, therapeutic acupuncture and conventional treatment in alleviating psychological distress in primary care patients follow up from an open, pragmatic randomized controlled trial

Bae, $\mathrm{H}$

Bae, $\mathrm{H}$

Min, B-II

Cho, S

Goyatá, SLT

Avelino, CCV

Santos, SVM

Souza Junior, DI

Gurgel, MDSL

Terra, FS

Lorent, L
Efficacy of Acupuncture in Reducing

Preoperative Anxiety: A Meta-Analysis

*Efeitos da acupuntura no tratamento da ansiedade: revisão integrativa.

Auricular Acupuncture Versus Progressive Muscle Relaxation in

uma paciente que

apresentava transtorno de ansiedade.

Analisar os artigos publicados no período de 2005-2010 que corresponde a implantação da

Revisão bibliográfica

Revisão de Literatura

Ensaio clínico randomizado

Ensaio clínico randomizado

Revisão de Literatura

Ensaio clínico randomizado

Revisão de Literatura

Revisão integrativa

Ensaio clínico prospectivo
Investigar se a acupuntura pode melhorar a memória e reduzir a ansiedade.

Avaliar e comparar os efeitos de um tratamento integrativo (TI), acupuntura terapêutica (TA) e tratamento convencional (CT) no alívio de sintomas de ansiedade e depressão em pacientes de cuidados primários psicologicamente angustiados

Identificar evidências na literatura sobre o efeito de orelha em protocolo de ansiedade e tratamento

Avaliar e comparar os efeitos de 6 meses de 8 semanas de um tratamento integrativo ( $\mathrm{TI})$, acupuntura terapêutica (TA) e tratamento convencional (CT) na redução dos sintomas de ansiedade, depressão e na melhoria da qualidade de vida relacionada à saúde (QVRS) e senso de coerência (SOC) em pacientes de atenção primária psicologicamente angustiados.

Avaliar a eficácia ansiolítica pré-operatória da terapia de acupuntura, este estudo conduziu uma meta-análise de uma série de estudos apropriados.

Avaliar as evidências científicas disponíveis na literatura sobre os efeitos da acupuntura no tratamento da ansiedade e a qualidade desses estudos.

Comparar a eficácia do $A A$ versus relaxamento muscular progressivo (PMR), um 


Agorastos, A
Yassouridis, A
Kellner, M
Muhtz, C
Klausenitz, C
Hacker, H
Hesse, T
Kohlmann, T
Endlich, K
Hahnenkamp, K

et al.

Ahlberg, $R$

Skårberg, $\mathrm{K}$

Brus, O

Kjellin, $\mathrm{L}$

Ormsby, SM

Smith, CA

Dahlen, HG

Hay, PJ

Lind, JM

Wiles, MD

Mamdani, J

Pullman, $\mathrm{M}$

Andrzejowski, JC

Kurebayashi, LFS

Turrini, RNT

Souza, TPB

Marques, CF

Rodrigues, RTF

Charlesworth, $\mathrm{K}$

Mafetoni, RR

Rodrigues, $\mathrm{MH}$

Jacob,LMS

Shimo, AKK

Silva, MT

Da Silva, EN

Barreto, JOM

Smith, CA

Lacey, S

Chapman, M

Ratcliffe, J

Norman, RJ

Johnson, NP, et al.

\section{Patients with Anxiety \\ Disorders or Major \\ Depressive Disorder: A \\ Prospective Parallel \\ Group Clinical Trial}

Auricular Acupuncture

for Exam Anxiety in

Medical Students-A

Randomized Crossover Investigation

Auricular acupuncture for substance use: a randomized controlled trial of effects on

anxiety, sleep, drug use and use of addiction treatment services

Evaluation of an antenatal acupuncture intervention as an adjunct therapy for antenatal depression (AcuAnteDep): study protocol for a pragmatic randomised controlled trial

A randomised controlled trial examining the effect of acupuncture at the EX-HN3 (Yintang) point on pre-operative anxiety levels in neurosurgical patients

Auriculotherapy to reduce anxiety and pain in nursing professionals: a randomized clinical trial

Effectiveness of auriculotherapy on anxiety during labor: a randomized clinical trial

Rapid response in health technology assessment: a Delph study for a Brazilian guideline

The effects of acupuncture on the secondary outcomes of anxiety and quality of

life for women

undergoing IVF: A

randomized controlled trial

em grupo

paralelo

método de relaxamento padronizado e aceito.

Investigação

cruzada

aleatória

randomizado

Ensaio clínico randomizado

Ensaio randomizado controlado

Ensaio clínico randomizado

Ensaio clínico randomizado

Revisão sistemática de Literatura

Ensaio randomizado controlado
Investigar se a acupuntura auricular (AA) pode reduzir a ansiedade do exame em comparação com o placebo e sem intervenção.

Avaliar o efeito de curto e longo prazo da acupuntura auricular na ansiedade, sono, uso de drogas e dependência utilização de tratamento em adultos com abuso de substâncias.

Investigar esta possibilidade de tratamento, com um exame adicional de um potencial efeito biomecanístico da acupuntura.

Determinar o efeito da acupuntura no EX-HN3 (ponto Yintang) nos níveis de ansiedade pré-operatória em pacientes neurocirúrgicos.

Avaliar a eficácia do protocolo auricular (APPA) na redução

da dor e ansiedade e na melhoria da qualidade de vida da equipe de enfermagem de um hospital.

Avaliar a eficácia da auriculoterapia na ansiedade da mulher durante o trabalho de parto.

A eficácia/efetividade e a segurança da acupuntura e da auriculoterapia para o tratamento da ansiedade ou depressão em adultos e/ou idosos.

Examinar os efeitos da acupuntura em comparação com sham acupuntura na qualidade de vida e ansiedade para mulheres submetidas a um ciclo de fertilização in vitro. 


\begin{tabular}{|c|c|c|c|c|}
\hline $\begin{array}{l}\text { Fleckenstein, J } \\
\text { Peter Krüger, P } \\
\text { Ittner, KP }\end{array}$ & 2018 & $\begin{array}{l}\text { Effects of single-point } \\
\text { acupuncture (HT7) in } \\
\text { the prevention of test } \\
\text { anxiety: Results of a } \\
\text { RCT }\end{array}$ & $\begin{array}{l}\text { Randomizado } \\
\text { controlado por } \\
\text { placebo }\end{array}$ & $\begin{array}{l}\text { Reproduzir os efeitos } \\
\text { descritos da acupuntura no } \\
\text { HT7 sobre a ansiedade de } \\
\text { forma padronizada. }\end{array}$ \\
\hline $\begin{array}{l}\text { Zeng, L } \\
\text { Tao, Y } \\
\text { Hou, W } \\
\text { Zong, L } \\
\text { Yu, L }\end{array}$ & 2018 & $\begin{array}{c}\text { Electro-acupuncture } \\
\text { improves psychiatric } \\
\text { symptoms, anxiety and } \\
\text { depression in } \\
\text { methamphetamine } \\
\text { addicts during } \\
\text { abstinence A } \\
\text { randomized controlled } \\
\text { trial }\end{array}$ & $\begin{array}{l}\text { Ensaio clínico } \\
\text { randomizado }\end{array}$ & $\begin{array}{l}\text { Observar o efeito da } \\
\text { eletroacupuntura na melhora } \\
\text { dos sintomas psiquiátricos, } \\
\text { bem como ansiedade e } \\
\text { depressão em viciados em } \\
\text { metanfetaminas (MA) durante } \\
\text { a abstinência usando ensaios } \\
\text { clínicos randomizados. }\end{array}$ \\
\hline $\begin{array}{l}\text { Novak, VC } \\
\text { Tilpp, SM } \\
\text { Bim, CR } \\
\text { Carrasco, AC }\end{array}$ & 2019 & $\begin{array}{l}\text { Effect of Acupuncture } \\
\text { on Improving Anxiety, } \\
\text { Sleep, and Quality of } \\
\text { Life }\end{array}$ & $\begin{array}{l}\text { Ensaio clínico } \\
\text { não } \\
\text { randomizado }\end{array}$ & $\begin{array}{l}\text { Verificar o efeito da } \\
\text { acupuntura na ansiedade, no } \\
\text { sono e na melhora da } \\
\text { qualidade de vida de } \\
\text { indivíduos ansiosos. }\end{array}$ \\
\hline $\begin{array}{c}\text { Zhaoa, B } \\
\text { Lia, Z } \\
\text { Wangc, Y } \\
\text { Mad, X } \\
\text { Wange, X } \\
\text { Wangf, X, et al. }\end{array}$ & 2019 & $\begin{array}{c}\text { Can acupuncture } \\
\text { combined with SSRIs } \\
\text { improve clinical } \\
\text { symptoms and quality of } \\
\text { life in patients with } \\
\text { depression? Secondary } \\
\text { outcomes of a } \\
\text { pragmatic randomized } \\
\text { controlled trial }\end{array}$ & $\begin{array}{l}\text { Ensaio clínico } \\
\text { randomizado }\end{array}$ & $\begin{array}{c}\text { Explorar os efeitos da } \\
\text { acupuntura (acupuntura } \\
\text { manual ou eletroacupuntura) } \\
\text { combinada com SSRIs para } \\
\text { depressão moderada a grave, } \\
\text { melhorando os principais } \\
\text { sintomas clínicos e a } \\
\text { qualidade de vida dos } \\
\text { pacientes em resultados } \\
\text { secundários. }\end{array}$ \\
\hline $\begin{array}{l}\text { Feng, J } \\
\text { Wang, W } \\
\text { Zhong, Y } \\
\text { Xing,C } \\
\text { Guo, T }\end{array}$ & 2019 & $\begin{array}{c}\text { Acupuncture for } \\
\text { perimenopausal } \\
\text { depressive disorder A } \\
\text { systematic review and } \\
\text { meta-analysis protocol }\end{array}$ & $\begin{array}{l}\text { Revisão } \\
\text { sistemática de } \\
\text { Literatura e } \\
\text { meta análise }\end{array}$ & $\begin{array}{c}\text { Explorar a eficácia e } \\
\text { segurança da acupuntura } \\
\text { para transtorno depressivo } \\
\text { perimenopausal (PDD). }\end{array}$ \\
\hline $\begin{array}{c}\text { Tu, C } \\
\text { MacDonald, I } \\
\text { Chen, Y }\end{array}$ & 2019 & $\begin{array}{c}\text { The Effects of } \\
\text { Acupuncture on } \\
\text { Glutamatergic } \\
\text { Neurotransmission in } \\
\text { Depression, Anxiety, } \\
\text { Schizophrenia, and } \\
\text { Alzheimer's Disease: A } \\
\text { Review of the Literature }\end{array}$ & $\begin{array}{c}\text { Revisão de } \\
\text { Literatura }\end{array}$ & $\begin{array}{l}\text { Nesta revisão, investigamos } \\
\text { as evidências atuais da } \\
\text { eficácia do tratamento da } \\
\text { acupuntura na depressão, } \\
\text { ansiedade, esquizofrenia e } \\
\text { Doença de Alzheimer (DA). }\end{array}$ \\
\hline
\end{tabular}

Silva, $\mathrm{HL}$

Almeida, MVS

Diniz, JSP

Leite,FMC

Moura, MAV

Bringuente,

MEO

Souza,CB et al.

Efeitos da

auriculoterapia na ansiedade de gestantes no pré-natal de baixo risco

\footnotetext{
Tong, $\mathrm{Q}$

Liu, $\mathrm{R}$

Zhang, K

Gao, $Y$

Cui, G

Shen, W

Tu, M

Jiang, $Y$

$\mathrm{Yu}, \mathrm{J}$

Liao, B

Fang, J
}

\section{Can acupuncture therapy reduce preoperative anxiety? A systematic review and meta-analysis}

Acupuncture for treating chronic stable angina anxiety and depression A protocol for pectoris-associated
Estudo clínico randomizado

Revisão sistemática de Literatura

Revisão sistemática de Literatura e meta análise
Avaliar os efeitos da auriculoterapia nos níveis de ansiedade em gestantes atendidas em pré-natal de baixo risco.

Avalia sistematicamente a eficácia e segurança da terapia de acupuntura para ansiedade pré-operatória, bem como a qualidade das evidências que apoiam esta aplicação.

Avaliar se a acupuntura é eficaz como terapia complementar para ansiedade e depressão em 


\begin{tabular}{|c|c|c|c|c|}
\hline & & $\begin{array}{l}\text { systematic review and } \\
\text { meta-analysis }\end{array}$ & & $\begin{array}{c}\text { pacientes com angina de } \\
\text { peito estável crônica (CSAP). }\end{array}$ \\
\hline $\begin{array}{l}\text { Tan, A } \\
\text { Wang, M } \\
\text { Liu, J } \\
\text { Huang, K } \\
\text { Dai, D } \\
\text { Li, L, et al. }\end{array}$ & 2020 & $\begin{array}{c}\text { Efficacy and safety of } \\
\text { acupuncture combined } \\
\text { with western medicine } \\
\text { for anxiety A systematic } \\
\text { review protocol }\end{array}$ & $\begin{array}{l}\text { Revisão } \\
\text { sistemática e } \\
\text { protocolo de } \\
\text { meta-análise }\end{array}$ & $\begin{array}{c}\text { Avaliar a eficácia e segurança } \\
\text { do tratamento combinado de } \\
\text { acupuntura e medicamentos } \\
\text { ocidentais. }\end{array}$ \\
\hline
\end{tabular}

Os resultados mostraram que a maior parte dos artigos publicados $(64,5 \%)$ no período analisado, ou seja, 2010 a 2020 foram realizadas nos últimos quatro anos. Com isso, a partir de 2016 houve um salto nas publicações, passando de uma média de 1 a 2 artigos por ano, para quatro publicações por ano, sobre a acupuntura como tratamento promissor para a ansiedade. Outro ponto a destacar no período analisado é o aumento de estudos clínicos $(60,8 \%)$ com relação às revisões de literatura $(39,2 \%)$ sobre o tema.

Esta revisão identificou alguns benefícios da acupuntura, eletroacupuntura e auriculoterapia no tratamento de ansiedade e depressão. De um modo geral, os tratamentos da Medicina Tradicional Chinesa trazem mais benefícios nos grupos estudados, principalmente nos experimentos de longo prazo, demonstrando ser esta uma possibilidade terapêutica para o tratamento de pessoas com ansiedade.

Dos artigos analisados, foi exposto que os protocolos resultaram em uma avaliação positiva com a redução da ansiedade, diminuição parcial dos sintomas, relato do alívio dos sintomas a partir de sessões consecutivas e que, tratamentos combinados podem melhorar os sintomas e a qualidade de vida de pacientes com depressão moderada a grave.

Os estudos também alertam para um aumento das amostras e de tempo em estudos posteriores, pois por mais que a terapia com acupuntura pode ser capaz de diminuir a ansiedade em pacientes com ansiedade, os resultados precisam ser mais verificados devido ao pequeno tamanho das amostras e à baixa qualidade das evidências até o momento.

\section{CONSIDERACOESS FINAIS}

Os trabalhos encontrados na revisão sistemática de literatura expuseram que há um efeito benéfico da acupuntura no tratamento dos sintomas da ansiedade e abre novas perspectivas para o tratamento de outros transtornos mentais e consequentemente dos físicos por meio de técnicas terapêuticas complementares, entre elas a acupuntura. 
Segundo Campiglia ${ }^{9}$ (2004) e Ross ${ }^{14}$ (2003), o tratamento realizado por meio da acupuntura não proporciona milagres ou o fim total das doenças nos pacientes. De modo processual, o tratamento por meio da acupuntura colabora com o restabelecimento da saúde e está diretamente relacionado a condições ambientais, climáticas, sociais e históricas, além de como o indivíduo cuida da alimentação, estados emocionais e da sua espiritualidade.

Apesar dessas descobertas, fazem-se necessários mais estudos clínicos e sistematizados, com amostras maiores através de pesquisas básicas e clínicas para mostrar a eficácia ou não desses procedimentos, possibilitando assim, que os portadores de ansiedade tenham acesso aos benefícios das técnicas terapêuticas complementares, como a acupuntura.

\section{REFERÉNCIAS}

1. Prado JM, Kurebayashi LFS, Silva MJP. Eficácia da auriculoterapia na redução de ansiedade em estudantes de enfermagem. Rev. esc. enferm. 2012; 46 (5):1200-1206.

2. Silva, ALP. O tratamento da ansiedade por intermédio da acupuntura: um estudo de caso. Psicol. cienc. prof., 2010; 30(1): 200-211.

3. Rocha, SV, Almeida, MMG, Araújo, TM, Virtuoso, J. J. S. Prevalência de transtornos mentais comuns entre residentes em áreas urbanas de Feira de Santana, Bahia. Revista Brasileira de Epidemiologia, 2010;13(4): 630-640.

4. Chade, J. Brasil é o país mais depressivo da América Latina, diz OMS. Gazeta do Povo, 2017. Disponível em: http:// saude.estadao.com.br/noticias/geral,brasil-e-o-pais-quemais-sofre-com-depressao-na-america-latina,70001676638 -20172021

5. Zatesko, P, Ribas-Silva, RC. Eficácia da Acupuntura no Tratamento de Ansiedade e Estresse Psicológico. Rev Bras Terap e Saúde, 2016; 6(2): 7-12.

6. Ministério da Saúde (Brasil). Política Nacional de Práticas Integrativas e Complementares no SUS - PNPIC-SUS / Ministério da Saúde, Secretaria de Atenção à Saúde, Departamento de Atenção Básica. - Brasília: Ministério da Saúde, 2006.

7. Yamamura, Y. Acupuntura Tradicional: A Arte de Inserir. 2. ed. São Paulo: Roca; 2001. 
8. Maciocia G. Os fundamentos da medicina chinesa: um texto abrangente para acupunturistas e fitoterapeutas. 2a ed. São Paulo: Roca; 2007.

9. Campiglia, H. Psique e Medicina Tradicional Chinesa. São Paulo: Roca; 2004.

10. Pieta, J, Leite, LL. Acupuntura no tratamento de ansiedade na Unidade Básica de Saúde Hélio Figueiredo em Bom Jesus-PI: uma proposta de intervenção. 2019; 1: 1-11.

11. Zotelli, VLR; Meirelles, MPMR; De Sousa, MLR. Uso da acupuntura no manejo da dor em pacientes com alterações na articulação temporomandibular (ATM). Revista de Odontologia da Universidade Cidade de São Paulo, 2017; 22 (2):185-188.

12. Alvarenga, TF, Amaral, CG, Steffen, CP. Ação da acupuntura na neurofisiologia da dor: revisão bibliográfica. Revista Amazônia Science \& Health. 2014; 2 (4): 29-36.

13. Santos, DR, Sperotto, DF, Pinheiro, UMS. A medicina tradicional chinesa no tratamento do transtorno de ansiedade: Um Olhar Sobre o Stress. Revista Contexto \&amp; Saúde, 2013; 11 (20): 103-112.

14. ROSS, J. Combinações dos Pontos de Acupuntura: A Chave para o Êxito. São Paulo: Roca; 2003.

15. Focks, C. Atlas de acupuntura: com sequência de fotos e ilustrações, textos didáticos e indicações clínicas. Barueri, SP: Manole; 2005. 